\title{
DKC1 serves as a potential prognostic biomarker for human clear cell renal cell carcinoma and promotes its proliferation, migration and invasion via the NF- $\mathrm{KB}$ pathway
}

\author{
MENG ZHANG $^{1 *}$, YU PAN $^{1 *}$, RANRAN JIANG $^{1,3^{*}}$, PINGFU HOU $^{1,2}$, HAIXIA SHAN $^{1,3}$, \\ FANG CHEN $^{1}$, TAO JIANG ${ }^{1}$, JIN BAI ${ }^{1,2}$ and JUNNIAN ZHENG ${ }^{1-3}$ \\ ${ }^{1}$ Cancer Institute and ${ }^{2}$ Jiangsu Center for the Collaboration and Innovation of Cancer Biotherapy, Xuzhou Medical University; \\ ${ }^{3}$ Center of Clinical Oncology, Affiliated Hospital of Xuzhou Medical University, Xuzhou, Jiangsu 221002, P.R. China
}

Received November 24, 2017; Accepted May 31, 2018

DOI: 10.3892/or.2018.6484

\begin{abstract}
DKC1, an X-linked gene encoding dyskerin at $\mathrm{Xq} 28$, is a crucial component of the telomerase complex and is indispensable for normal telomere function and the posttranscriptional modification of precursor rRNA. It has been revealed to exert diverse biological functions and prognostic values in numerous types of cancers. Our present study was aimed at examining DKC1 expression in normal renal tissues and clear cell renal cell carcinoma (ccRCC) samples and the prognostic value of DKC1 in ccRCC. We examined DKC1 protein expression levels in tissue microarrays including 307 cases of ccRCC tissues and in 75 pairs of ccRCC and paracancerous tissues with immunohistochemistry. The percentage of $\mathrm{DKC1}$ expression in ccRCC $(61.3 \%)$ was markedly higher than that in paracancerous tissues $(34.7 \%)(\mathrm{P}=0.001)$. Positive $\mathrm{DKC} 1$ expression tends to significantly be associated with unfavorable clinicopathological characteristics such as tumor diameter $>7 \mathrm{~cm}(\mathrm{P}=0.002)$ and tumor-node-metastasis (TNM) stage III or IV $(\mathrm{P}<0.001)$. Multivariate COX analysis revealed that positive DKC1 expression was an independent unfavorable factor for prognosis of ccRCC patients [hazard ratio $(\mathrm{HR})=1.932,95 \% \mathrm{CI}$,
\end{abstract}

Correspondence to: Professor Jin Bai or Professor Junnian Zheng, Cancer Institute, Xuzhou Medical University, 84 West Huaihai Road, Xuzhou, Jiangsu 221002, P.R. China

E-mail: bj@xzhmu.edu.cn

E-mail: jnzheng@xzhmu.edu.cn

*Contributed equally

Abbreviations: ccRCC, clear cell renal cell carcinoma; IHC, immunohistochemistry; NF- $\kappa \mathrm{B}$, nuclear factor $\kappa \mathrm{B}$; MMPs, matrix metalloproteinases; TMA, tissue microarray; IRS, immunoreactive score; TCGA, The Cancer Genome Atlas; GAPDH, glyceraldehyde3-phosphate dehydrogenase; ECM, extracellular matrix; HR, hazard ratio; $\mathrm{CI}$, confidence interval

Key words: renal cell carcinoma, DKC1, NF- $\mathrm{BB}$, prognostic biomarker, tumorigenesis
1.290-2.893, $\mathrm{P}=0.001$ for 5-year overall survival; $\mathrm{HR}=1.778$, 95\% CI,1.150-2.748, $\mathrm{P}=0.010$ for disease-free survival]. In the PROGgeneV2 platform, we also found that ccRCC patients with high DKC1 mRNA expression had a poorer prognosis than patients with low DKC1 expression in The Cancer Genome Atlas (TCGA). Furthermore, we found that knockdown of $\mathrm{DKC1}$ inhibited proliferation, migration and invasion of ccRCC through regulation of the $\mathrm{NF}-\kappa \mathrm{B} / \mathrm{MMP}-2$ signaling pathway in vitro. We also demonstrated that $\mathrm{DKC} 1$ regulated ccRCC proliferation and the expression of NF- $\kappa \mathrm{B}-\mathrm{p} 65$ and MMP-2 in vivo. In summary, the expression of DKC1 was upregulated in ccRCC, which was associated with unfavorable clinicopathological characteristics and DKC1 may act as an independent prognostic indicator of ccRCC patients.

\section{Introduction}

Renal cell carcinoma (RCC) is accountable for $\sim 90 \%$ of renal cancer patients and is the most prevalent malignancies of kidney cancer (1). Among all RCC histological subtypes, clear cell RCC (ccRCC) is the most malignant form and leads to the most cancer-related deaths (2). Although marked development has been achieved in the diagnosis and treatment of ccRCC, $\sim 30 \%$ of patients have metastatic disease (3). Surgery is still the most effective treatment for localized primary ccRCC, but ccRCC is resistant to conventional treatments such as radiation, hormone treatment and chemotherapy. Despite the fact that specific targeted therapies with favorable clinical outcomes to a certain extent have been developed, individual differences in response and the risk of adverse effects restrict the use of these drugs (4). Therefore, appropriate ccRCC biomarkers may contribute to improve early diagnosis and patient therapy.

Dyskerin is a predominantly nucleolar protein encoded by the $\mathrm{DKC1}$ gene and is involved in dyskeratosis congenital (5). It is a component of H/ACA small nucleolar ribonucleoprotein with various characteristics, such as bone marrow failure, mucocutaneous abnormalities and an increased tumor susceptivity (6). Several lines of evidence have demonstrated that DKC1 expression is significantly upregulated and associated with poor prognosis in some human cancers, such as prostate cancer (7), neuroblastomas (8) and hepatocellular carcinoma (9). Despite 
these survey results, the significance of dyskerin expression in cancer has been disputed, with some research revealing that dyskerin may have a function as a tumor suppressor $(10,11)$. In addition, the specific mechanism and significance of $\mathrm{DKC1}$ in ccRCC progression are not fully known.

In this study, we sought to examine the expression levels of DKC1 in ccRCC, and then explored its association with the clinical characteristics, 5-year overall and disease-specific survival of ccRCC patients. In addition, we explored the difference in the expression levels of $\mathrm{DKC1}$ in renal cancer and paracancerous tissues. Moreover, we also investigated how DKC1 regulated ccRCC cell proliferation, migration and invasion in vitro, and tumor growth in vivo as well as the molecular mechanisms.

\section{Materials and methods}

Patients and specimens. Two independent retrospective ccRCC cohorts with tissue microarrays (TMAs) were examined in the present study. The small ccRCC TMA was obtained from Shanghai Outdo Biotechnology (Shanghai, China), which contained 75 pairs of ccRCC tissues and matched paracancerous tissues. It contained 75 patients who underwent radical nephrectomy between November 2006 and September 2008. Another large TMA was composed of 310 ccRCC tissues from patients collected from the Affiliated Hospital of Xuzhou Medical University who underwent radical nephrectomy without prior treatment from February 2005 to December 2008, and their clinicopathological characteristics containing age, sex, tumor diameter, depth of invasion, lymph node and distant metastasis, as well as tumor-node-metastasis (TNM) stage were obtained. Due to specimen deficiency during antigen retrieval, finally 307 ccRCC patients in the large TMA were utilized to explore the association of $\mathrm{DKC1}$ expression with clinicopathological features. Informed consents from all patients were obtained and institutional approval was obtained by the review board of the Affiliated Hospital of Xuzhou Medical University prior to this study.

Construction of TMAs and immunohistochemistry (IHC). Construction of the large TMA was performed by contract service at the National Engineering Center for Biochip (Shanghai, China). Each tissue microarray dot was cut $1.5 \mathrm{~mm}$ in diameter from the paraffin tumor block. The standard procedure for IHC of TMA was performed as previously described (12). The polyclonal rabbit anti-DKC1 (1:50, cat. no. ab64667; Abcam, Cambridge, MA, USA) was used for primary antibody incubation at $4^{\circ} \mathrm{C}$ overnight. The slides without primary antibody incubation were used as negative controls.

Assessment of immunostaining. The evaluation of DKC1 staining was blindly examined by two pathologists. Positive DKC1 immunostaining was defined mainly in the cytoplasm but could also be observed in the nucleus area. The tissues were scored according to both the intensity and percentage of cells with positive staining. The staining intensity of DKC1 was scored as $0-3$ ( $0=$ negative; $1=$ weak; $2=$ moderate; $3=$ strong). The percentage of DKC1-positive stained cells was also scored into 4 categories: 1 (0-25\%); 2 (26-50\%); 3 (51-75\%); and $4(76-100 \%)$. In the case of a discrepancy between copied cores, the mean score from the two tissue cores was selected as the final score. The level of DKC1 staining was evaluated by immunoreactive score (IRS), which is calculated by multiplying the scores of the staining intensity and the percentage of positive cells. For statistical analysis, scores of 0-5 were considered as low expression, while scores of 6-12 were considered as high expression; the cut-off value was determined by receiver operating characteristic curve analysis.

Animals and cell lines. A total of 20 female BALB/c nude mice weighing 14-18 g, 6-8 weeks old, were purchased from Beijing HFK Bioscience Co., Ltd. (Beijing, China) for studies approved by the Animal Care Committee of Xuzhou Medical University and maintained under specific pathogen-free conditions. Human embryo kidney epithelial cell line HK-2 and human ccRCC cell lines ACHN, 786-O and OSRC-2 were purchased from the Shanghai Institute of Biochemistry and Cell Biology, Chinese Academy of Sciences (Shanghai, China). HK-2 cells were cultured in keratinocyte serum free medium (K-SFM) supplemented with $10 \%$ fetal calf serum (FCS; both from Invitrogen; Thermo Fisher Scientific, Inc., Shanghai, China). ACHN cells were cultured in Minimum Essential Media medium (MEM) supplemented with $10 \%$ FCS (both from Invitrogen; Thermo Fisher Scientific, Inc.). 786-O and OSRC-2 cells were cultured in Roswell Park Memorial Institute-1640 medium (RPMI-1640; Invitrogen; Thermo Fisher Scientific, Inc., Shanghai, China) supplemented with $10 \%$ FCS. These four cell lines were both incubated in a $37^{\circ} \mathrm{C}$ humidified incubator with $5 \% \mathrm{CO}_{2}$.

Plasmid, siRNA and shRNA transfections. The DKC1 siRNA and scrambled siRNA were purchased from Shanghai GenePharma Co., Ltd., (Shanghai, China). The p65 overexpression plasmids were obtained from $\mathrm{Dr} \mathrm{Yu}$ Wu (Nanjing Medical University, Nanjing, China). DKC1 siRNA and scrambled siRNA were transfected into the ACHN and 786-O cells by siLentFect ${ }^{\mathrm{TM}}$ Lipid reagent (Bio-Rad Laboratories, Hercules, CA, USA) following the manufacturer's protocol. Transfection of p65 overexpression plasmid and control vector into the $\mathrm{ACHN}$ and 786-O cells was performed using Lipofectamine 2000 transfection reagent (Invitrogen; Thermo Fisher Scientific, Inc.) according to the manufacturer's instructions. The DKC1 knockdown ACHN cell lines (DKC1 $\left.{ }^{\mathrm{KD}}-\mathrm{ACHN}\right)$ and control ACHN cell lines (Ctrl-ACHN) were established by transfecting with lentivirus packing DKC1 shRNA expression and control vector, respectively (Shanghai GenePharma). Target cells were transfected with the lentivirus for $48 \mathrm{~h}$ and then selected with puromycin (Santa Cruz Santa Cruz Biotechnology, Inc., Dallas, TX, USA) at a concentration of $5 \mu \mathrm{g} / \mathrm{ml}$ for 3 weeks.

Cell proliferation assay. Complete medium ( $2 \mathrm{ml})$ containing $1 \times 10^{5}$ stable DKC1 knockdown ACHN or $786-\mathrm{O}$ cells and corresponding controls were seeded in 6-well plates and cultured for 24, 48, 72 and $96 \mathrm{~h}$. At exact time-points, the number of cells in the DKC1 knockdown ACHN or 786-O cells and corresponding controls was counted respectively.

Cell migration and invasion assays. Cell migration and invasion assays were carried out using Transwell filter inserts (8.0- $\mu \mathrm{m}$ pore size with polycarbonate membranes) precoated with or without Matrigel (BD Biosciences, Franklin Lakes, NJ, USA). The detailed conditions were previously described (13). 
A

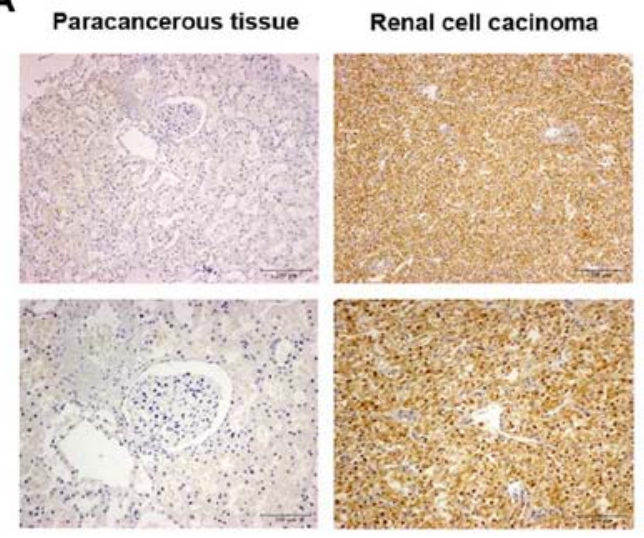

B

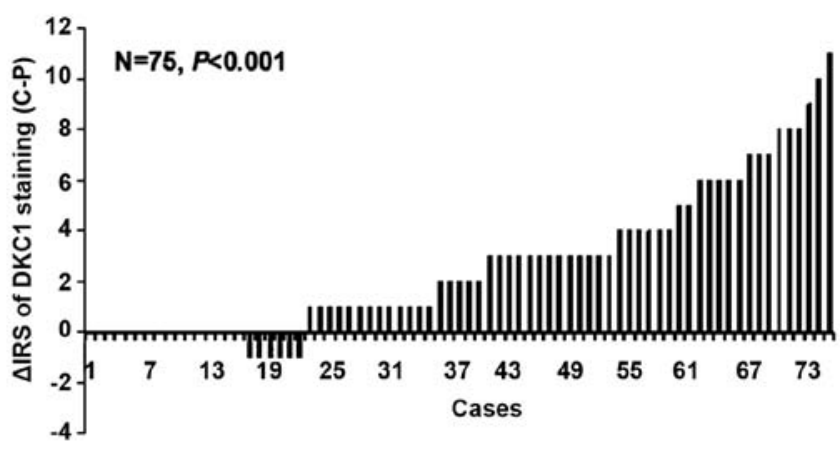

C
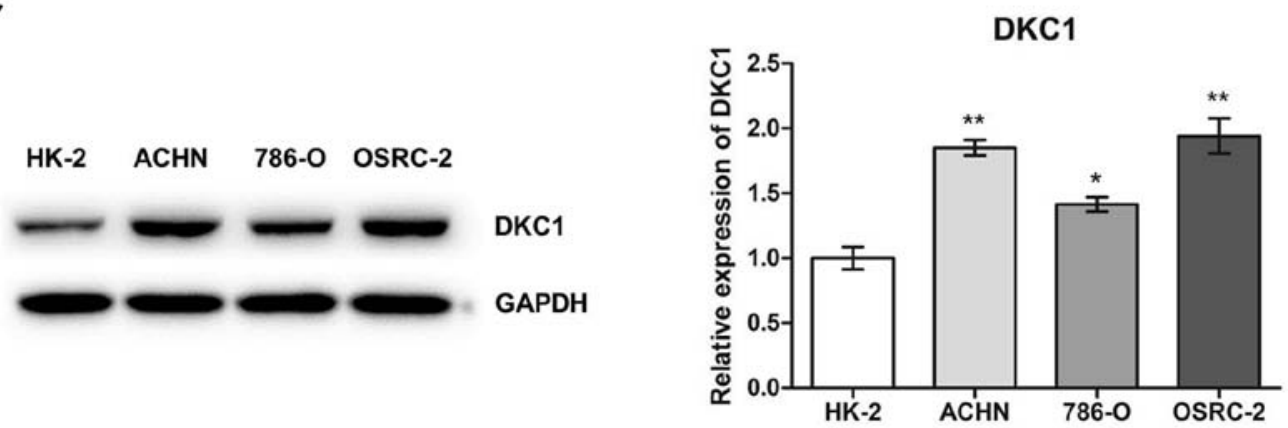

Figure 1. DKC1 expression is increased in ccRCC compared with normal renal tissues and cell lines. (A) Representative images of DKC1 immunohistochemical staining in TMA are shown. Top panel, magnification, x100; Bottom panel, magnification, x200. (B) The distribution of the difference in staining intensities of DKC1 in ccRCC tissues compared with paracancerous tissues. C, ccRCC tissues; P, paracancerous tissues; IRS, immunoreactivity score. (C) Western blot analysis of DKC1 expression in human embryo kidney epithelial cells HK-2 and renal cell carcinoma cell lines, including ACHN, 786-O an OSRC-2. Data are presented as the mean $\pm \mathrm{SD},{ }^{*} \mathrm{P}<0.05,{ }^{* *} \mathrm{P}<0.01$. ccRCC, clear cell renal cell carcinoma.

Antibodies and western blotting (WB). WB was performed as previously reported (14). The following antibodies against the corresponding proteins were used for WB: DKC1 $(1: 1,000$ for WB, cat. no. ab64667; Abcam); NF-kB-p65 (1:1,000 for WB; 1:50 for IHC, cat. no. 8242P; Cell Signaling Technology, Inc., Beverly, MA, USA); MMP-2 (1:1,000 for WB, 1:50 for IHC, cat. no. 4022S; Cell Signaling Technology, Inc.); p-AKT (1:1,000 for WB, cat. no. 4060T; Cell Signaling Technology, Inc.); p-S6K (1:1,000 for WB, cat. no. 9234T; Cell Signaling Technology, Inc.); MMP-9 (1:1,000 for WB, cat. no. 3852S; Cell Signaling Technology, Inc.) and GAPDH (1:5,000 for WB, cat. no. sc-365062; Santa Cruz Biotechnology, Inc.). Then, HRP-conjugated goat anti-mouse and goat anti-rabbit secondary antibodies (1:10,000, anti-mouse, cat. no. SA00001-1, anti-rabbit cat. no. SA00001-2; Proteintech Group, Inc., Rosemont, IL, USA) were applied to the blot for $1 \mathrm{~h}$ at room temperature.

Subcutaneous tumor model in vivo. To produce a subcutaneous tumor model in vivo, the BALB/c nude mice were randomly divided into two groups consisting of 15 mice each. Stable DKC1 knockdown $\left(1 \times 10^{6}\right)$ and control ACHN cells were suspended in $200 \mu$ l phosphate-buffered saline (PBS) and subcutaneously injected through axillary fossa, respectively. After 2 weeks, the two groups of mice were sacrificed; their subcutaneous tumors were excised and fixed in $10 \%$ buffered formalin for statistical analysis and further histopathological analysis.
Statistical analysis. Paired Wilcoxon test was used to explore the difference of DKC1 staining between tumors and their matched non-tumor tissues. Fisher's exact test was carried out to assess the correlation between DKC1 expression and clinicopathological features. The Kaplan-Meier method with a log-rank test was used to investigate the differences of the 5-year survival and disease-specific survival between the positive $\mathrm{DKC} 1$ expression group and the negative expression group. Univariate and multivariate Cox proportional hazards regression analysis were used to evaluate the hazard ratios (HRs) and $95 \%$ confidence interval (CI) of HRs. Independent samples t-test was used in the proliferation, migration and invasion assays. All the statistical analyses were performed by SPSS 20.0 statistical software package (IBM Corp., Armonk, NY, USA). A P-value of $<0.05$ was defined as statistically significant, and all tests were two-sided.

\section{Results}

DKCl expression is increased in ccRCC tissues and RCC cell lines. To explore whether the expression level of DKC1 was altered in RCC, a small TMA was used to investigate DKC1 protein expression in 75 pairs of ccRCC and paracancerous tissues using IHC. Our data revealed that DKC1 protein was localized in both the cytoplasm and nucleolus (Fig. 1A). As shown in Table I, high DKC1 expression was detected in 46 out of $75(61.3 \%)$ ccRCC tissues and in 26 out of 75 (34.7\%) 
Table I. DKC1 expression in ccRCC and paracancerous tissues.

DKC1 staining

\begin{tabular}{|c|c|c|c|c|}
\hline \multirow[b]{2}{*}{ Tissues } & \\
\hline & Low (\%) & High (\%) & Total & P-value \\
\hline Paracancerous tissues & $49(65.3)$ & $26(34.7)$ & 75 & 0.001 \\
\hline Renal cell carcinoma & $29(38.7)$ & $46(61.3)$ & 75 & \\
\hline
\end{tabular}

${ }^{\mathrm{a}} \chi^{2}$ test. ccRCC, clear cell renal cell carcinoma.

Table II. DKC1 staining and clinicopathological characteristics of 307 renal cancer patients.

\begin{tabular}{|c|c|c|c|c|}
\hline \multirow[b]{2}{*}{ Variables } & \multicolumn{4}{|c|}{ DKC1 staining } \\
\hline & Low $(\%)$ & $\operatorname{High}(\%)$ & Total & P-value ${ }^{a}$ \\
\hline Age (years) & & & & 0.674 \\
\hline$\leq 56$ & $119(80.4)$ & $29(19.6)$ & 148 & \\
\hline$>56$ & $124(78.0)$ & $35(22.0)$ & 159 & \\
\hline Sex & & & & 0.460 \\
\hline Male & $164(80.4)$ & $40(19.6)$ & 204 & \\
\hline Female & $79(76.7)$ & $24(23.3)$ & 103 & \\
\hline Tumor size $(\mathrm{cm})$ & & & & 0.002 \\
\hline$\leq 7$ & $115(57.5)$ & $85(42.5)$ & 200 & \\
\hline$>7$ & $41(38.3)$ & $66(61.7)$ & 107 & \\
\hline pT status & & & & $<0.001$ \\
\hline $\mathrm{pT}_{1}-\mathrm{pT}_{2}$ & $178(74.8)$ & $60(25.2)$ & 238 & \\
\hline $\mathrm{pT}_{3}-\mathrm{pT}_{4}$ & $28(40.6)$ & $41(59.4)$ & 69 & \\
\hline pN status & & & & 0.041 \\
\hline $\mathrm{pN}_{0}$ & $154(54.0)$ & $131(46.0)$ & 285 & \\
\hline $\mathrm{pN}_{1}-\mathrm{pN}_{3}$ & $9(40.9)$ & $13(59.1)$ & 22 & \\
\hline pM status & & & & 0.609 \\
\hline $\mathrm{pM}_{0}$ & $166(57.6)$ & $122(42.4)$ & 288 & \\
\hline $\mathrm{pM}_{1}$ & $8(42.1)$ & $11(57.9)$ & 19 & \\
\hline TNM stage & & & & $<0.001$ \\
\hline I-II & $221(93.6)$ & $15(6.4)$ & 236 & \\
\hline III-IV & $22(31.0)$ & $49(69.0)$ & 71 & \\
\hline
\end{tabular}

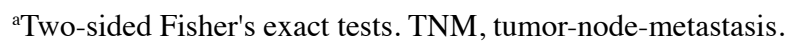

paracancerous tissues, and there was a significant expression difference between ccRCC and paracancerous tissues (Fig. 1B). Furthermore, western blot analysis revealed that the expression of DKC1 was significantly lower in HK-2, a type of human embryo kidney epithelial cell line, as compared with that in all 3 analyzed RCC cell lines, including ACHN, 786-O and OSRC-2 (Fig. 1C). Consequently, our results demonstrated that DKC1 was increased in RCC.

DKC1 expression is associated with clinicopathological characteristics in ccRCC patients. To further study the association between DKC1 expression and clinicopathological features, another TMA including 307 cases of ccRCC tissues was used to investigate DKC1 protein expression. There were 204 male and 103 female patients. Their mean age was 55.8 years. The distribution of the TNM stage was as follows: 181 patients at stage I, 55 at stage II, 40 at stage III and 31 at stage IV. Table II contains the association between DKC1 and the clinicopathological features of ccRCC patients. The data revealed that there were significant correlations of $\mathrm{DKC1}$ expression with tumor size $(\mathrm{P}=0.002)$, pT status $(\mathrm{P}<0.001)$, $\mathrm{pN}$ status $(\mathrm{P}=0.041)$ and TNM stage $(\mathrm{P}<0.001)$. However, no significance was found between DKC1 expression and other clinical characteristics, such as sex and age. These results indicated that high DKC1 expression tends to be associated with advanced clinicopathological parameters in ccRCC patients. 

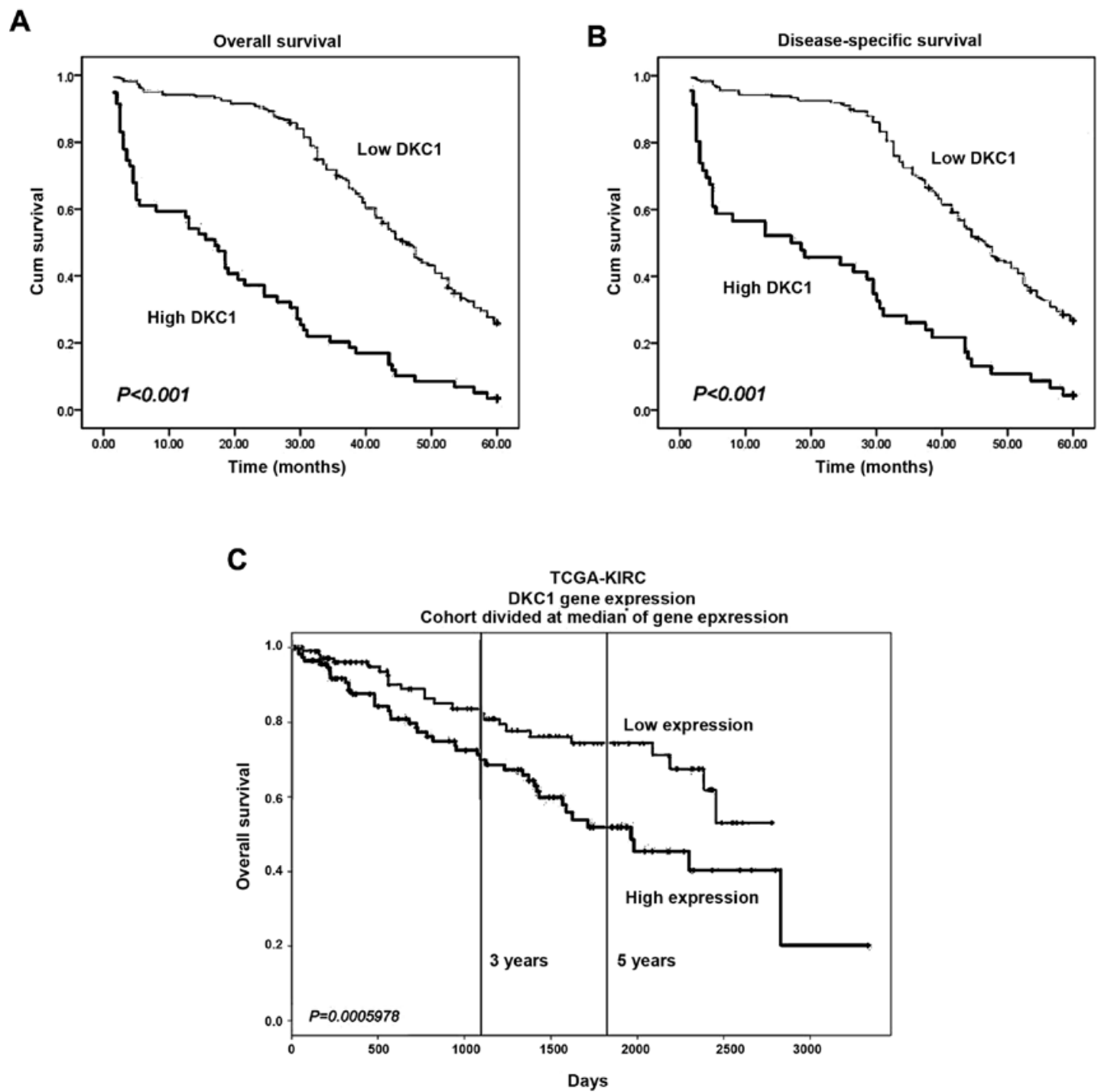

Figure 2. DKC1 expression is correlated with prognosis in ccRCC patients. (A and B) Kaplan-Meier curves revealed the difference of cum survival in the 5 -year overall survival and the disease-specific survival of the ccRCC patients with high/low DKC1 expression. (C) Increased DKC1 mRNA expression resulted in poor survival of ccRCC patients in TCGA. Survival data were searched for and analyzed from PROGgeneV2 (http://watson.compbio.iupui. edu/chirayu/proggene/database/?url=proggene). ccRCC, clear cell renal cell carcinoma; cum survival, cumulative survival; TCGA, The Cancer Genome Atlas.

DKC1 expression is correlated with the prognosis of ccRCC patients. To evaluate the prognostic value of DKC1 expression in ccRCC, the log-rank test combined with Kaplan-Meier survival curves was established. Our data revealed that ccRCC patients with high DKC1 expression were correlated with greater unfavorable 5-year overall and disease-specific survival than the rest of the patients with low DKC1 expression $(\mathrm{P}<0.001$ and $\mathrm{P}<0.001$, respectively) (Fig. 2A and B). Furthermore, to investigate whether DKC1 was an independent prognostic marker in ccRCC, univariate and multivariate COX analysis was performed. In the univariate $\mathrm{COX}$ analysis, our data suggested that increased DKC1 expression was significantly correlated with a worse 5-year overall and disease-specific survival, as well as with other prognostic markers, such as tumor size, $\mathrm{pT}$ status, $\mathrm{pN}$ status, pM status and TNM stage (Table III). In addition, both increased DKC1 expression (HR=1.932, 95\% CI, 1.290-2.893, $\mathrm{P}=0.001$ for 5 -year overall survival; $\mathrm{HR}=1.778,95 \% \mathrm{CI}$, $1.150-2.748, \mathrm{P}=0.010$ for disease-free survival) and TNM stage $(\mathrm{HR}=2.790,95 \% \mathrm{CI}, 1.861-4.184, \mathrm{P}<0.001$ for 5 -year overall survival; $\mathrm{HR}=2.689,95 \% \mathrm{CI}, 1.746-4.140, \mathrm{P}<0.001$ for disease-free survival) were independent adverse prognostic markers for the 5-year overall and disease-specific survival of ccRCC patients in multivariate COX analysis (Table IV). By searching the PROGgeneV2 platform, we found that ccRCC patients with high DKC1 expression had significant poorer prognosis than those with low DKC1 expression in The Cancer Genome Atlas (TCGA) which was consistent with our findings (Fig. 2C).

Knockdown of DKC1 suppresses ccRCC cell proliferation, migration and invasion in vitro. To explore the role of DKC1 in ccRCC progression, $\mathrm{ACHN}$ and 786-O cells were transiently transfected with control siRNA and DKC1 siRNA, respectively (Fig. 3A and B). Then the cell proliferation assays were carried out and the data revealed that DKC1 depletion led to a significant decrease in cell proliferation (Fig. 3C and D). Since high DKC1 expression was relevant 
Table III. Univariate Cox proportional regression analysis on the 5-year overall and disease-specific survival of ccRCC patients.

\begin{tabular}{|c|c|c|c|c|c|c|}
\hline \multirow[b]{2}{*}{ Variables } & \multicolumn{3}{|c|}{ Overall survival } & \multicolumn{3}{|c|}{ Disease-specific survival } \\
\hline & Hazard ratio & $95 \% \mathrm{CI}^{\mathrm{b}}$ & P-value ${ }^{a}$ & Hazard ratio & $95 \% \mathrm{CI}^{\mathrm{b}}$ & P-value ${ }^{a}$ \\
\hline DKC1 & & & & & & $<0.001$ \\
\hline Low & 1.000 & & $<0.001$ & 1.000 & & \\
\hline High & 3.525 & $2.574-4.828$ & & 3.126 & $2.213-4.414$ & \\
\hline Age (years) & & & & & & 0.728 \\
\hline$\leq 56$ & 1.000 & & 0.637 & 1.000 & & \\
\hline$>56$ & 1.067 & $0.810-1.410$ & & 1.053 & $0.786-1.411$ & \\
\hline Tumor size (cm) & & & & & & 0.021 \\
\hline$\leq 7$ & 1.000 & & 0.003 & 1.000 & & \\
\hline$>7$ & 1.676 & $1.194-2.352$ & & 1.549 & $1.069-2.244$ & \\
\hline pT status & & & & & & 0.014 \\
\hline $\mathrm{pT}_{1}-\mathrm{pT}_{2}$ & 1.000 & & 0.002 & 1.000 & & \\
\hline $\mathrm{pT}_{3}-\mathrm{pT}_{4}$ & 1.574 & $1.178-2.102$ & & 1.473 & $1.081-2.007$ & \\
\hline pN status & & & & & & $<0.001$ \\
\hline $\mathrm{pN}_{0}$ & 1.000 & & $<0.001$ & 1.000 & & \\
\hline $\mathrm{pN}_{1}-\mathrm{pN}_{3}$ & 3.421 & $1.842-6.352$ & & 3.046 & $1.686-5.502$ & \\
\hline pM status & & & & & & 0.021 \\
\hline $\mathrm{pM}_{0}$ & 1.000 & & $<0.001$ & 1.000 & & \\
\hline $\mathrm{pM}_{1}$ & 4.162 & $2.282-7.594$ & & 2.870 & $1.172-7.027$ & \\
\hline TNM stage & & & & & & $<0.001$ \\
\hline I-II & 1.000 & & $<0.001$ & 1.000 & & \\
\hline III-IV & 4.084 & $2.972-5.613$ & & 3.713 & $2.628-5.247$ & \\
\hline
\end{tabular}

${ }^{\text {aP}} \mathrm{P}$-values are from log-rank test; ${ }^{\mathrm{b}} \mathrm{CI}$, confidence interval. ccRCC, clear cell renal cell carcinoma; TNM, tumor-node-metastasis.

Table IV. Multvariate Cox regression analysis on the 5-year overall and disease-specific survival of ccRCC patients.

\begin{tabular}{|c|c|c|c|c|c|c|}
\hline \multirow[b]{2}{*}{ Variables $^{\mathrm{a}}$} & \multicolumn{3}{|c|}{ Overall survival } & \multicolumn{3}{|c|}{ Disease-specific survival } \\
\hline & Hazard ratio & $95 \% \mathrm{CI}^{\mathrm{b}}$ & P-value ${ }^{a}$ & Hazard ratio & $95 \% \mathrm{CI}^{\mathrm{b}}$ & P-value ${ }^{a}$ \\
\hline DKC1 & 1.932 & $1.290-2.893$ & 0.001 & 1.778 & $1.150-2.748$ & 0.010 \\
\hline Age (years) & 1.189 & $0.897-1.576$ & 0.228 & 1.126 & $0.838-1.513$ & 0.432 \\
\hline Tumor size & 1.319 & $0.930-1.871$ & 0.120 & 1.293 & $0.885-1.888$ & 0.184 \\
\hline TNM stage & 2.790 & $1.861-4.184$ & $<0.001$ & 2.689 & $1.746-4.140$ & $<0.001$ \\
\hline
\end{tabular}

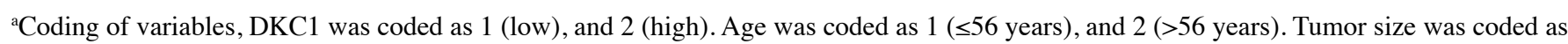
$1(\leq 7 \mathrm{~cm})$, and $2(>7 \mathrm{~cm})$. TNM stage was coded as 1 (I-II), and 2 (III-IV); ${ }^{\mathrm{b}} \mathrm{CI}$, confidence interval. ccRCC, clear cell renal cell carcinoma; TNM, tumor-node-metastasis.

to poor prognosis in the ccRCC patient cohort, we further explored the metastatic role of DKC1 in ccRCC cells. The Transwell assays were performed and our data revealed that knockdown of DKC1 decreased the abilities of cell migration in ACHN and 786-O cells when compared with the corresponding controls (Fig. 4A and B). In accordance with these consequences, the abilities of cell invasion were significantly decreased in ACHN and 786-O cells by DKC1 siRNAs when compared with the respective controls (Fig. 4C and D).
Knockdown of DKCl inhibits ccRCC cell proliferation, migration and invasion via regulation of the $N F-\kappa B / M M P-2$ signaling pathway. $\mathrm{NF}-\kappa \mathrm{B}$ is a crucial transcriptional factor and plays an important role in tumorigenesis. It regulates cell proliferation, metastasis, angiogenesis and survival, thus it is not surprising that $\mathrm{NF}-\kappa \mathrm{B}$ has been demonstrated to be activated in many human cancers (15). Its $\kappa \mathrm{B}$ site was found in the promoters of genes encoding MMP-2 which plays a critical role in cancer metastasis (16). In order to investigate the possible mechanism of $\mathrm{DKC} 1$ regulation of proliferation and metastasis in ccRCC 
A

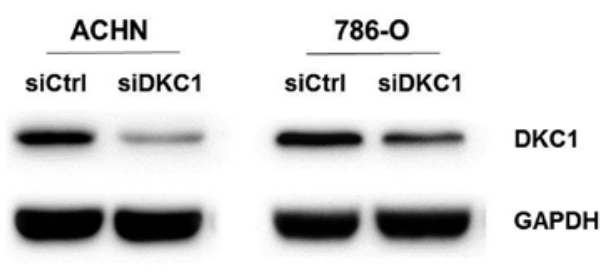

C

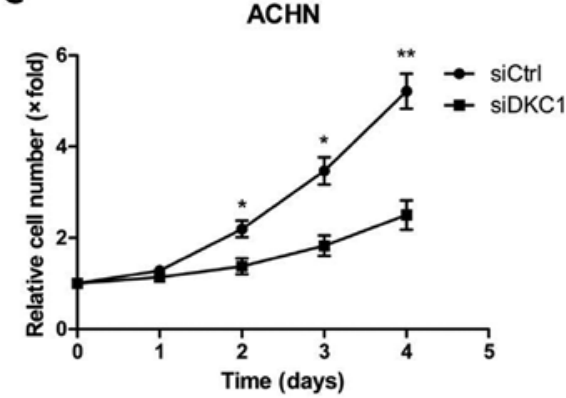

B

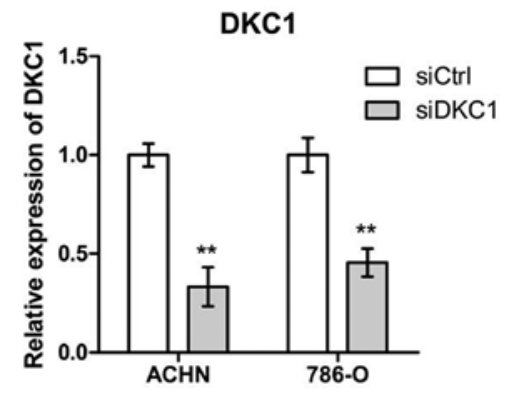

D

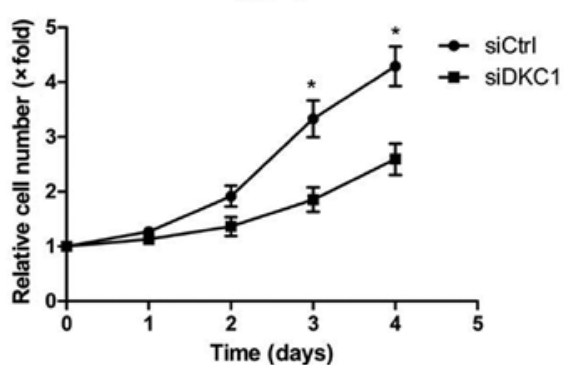

Figure 3. Knockdown of DKC1 inhibits cell proliferation of ccRCC cells in vitro. (A and B) Western blot analysis revealed that DKC1 expression in ACHN and 786-O was markedly knocked down by siRNA when compared with corresponding controls. (C and D) Cell proliferation assays were carried out to assess the cell proliferation rates of $\mathrm{ACHN}$ and $786-\mathrm{O}$ cells with different $\mathrm{DKC} 1$ expression levels. Data are presented as the mean $\pm \mathrm{SD},{ }^{*} \mathrm{P}<0.05,{ }^{* *} \mathrm{P}<0.01$. ccRCC, clear cell renal cell carcinoma.

A

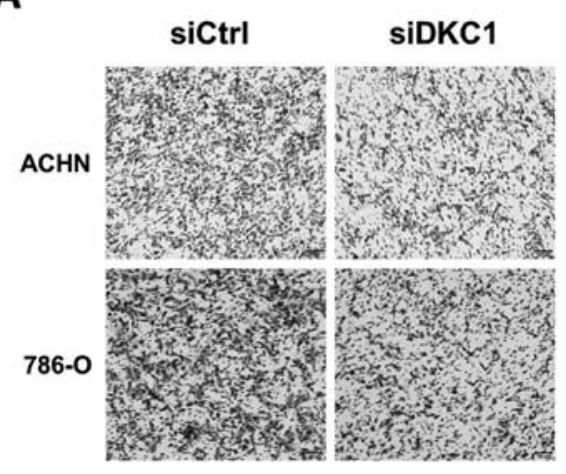

B

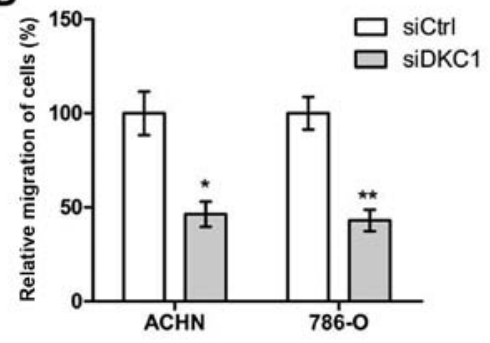

C

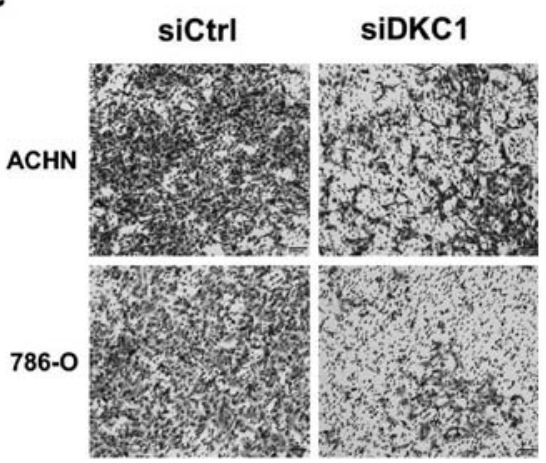

D

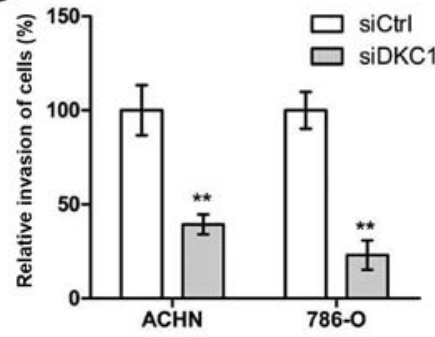

Figure 4. Knockdown of DKC1 inhibits the migration and invasion of ccRCC cells in vitro. (A) The migration of ACHN and 786-O cells with different DKC1 expression levels. (B) The cell migration number per field was counted in three random fields in ACHN and 786-O cells. (C) The invasion of ACHN and 786-O cells with different DKC1 expression levels. (D) The cell invasion number per field was counted in three random fields in ACHN and 786-O cells. Data are presented as the mean $\pm \mathrm{SD},{ }^{*} \mathrm{P}<0.05,{ }^{* *} \mathrm{P}<0.01$. ccRCC, clear cell renal cell carcinoma.

cells, we carried out western blotting to explore the protein levels of NF- $\kappa$ B and MMPs in ACHN and 786-O cells. Our data revealed that DKC1 knockdown significantly inhibited p65 and MMP-2 protein expression in ACHN and 786-O cells compared with the corresponding controls, but not MMP-9 (Fig. 5A). Since the PI3K/AKT pathway is also an important 

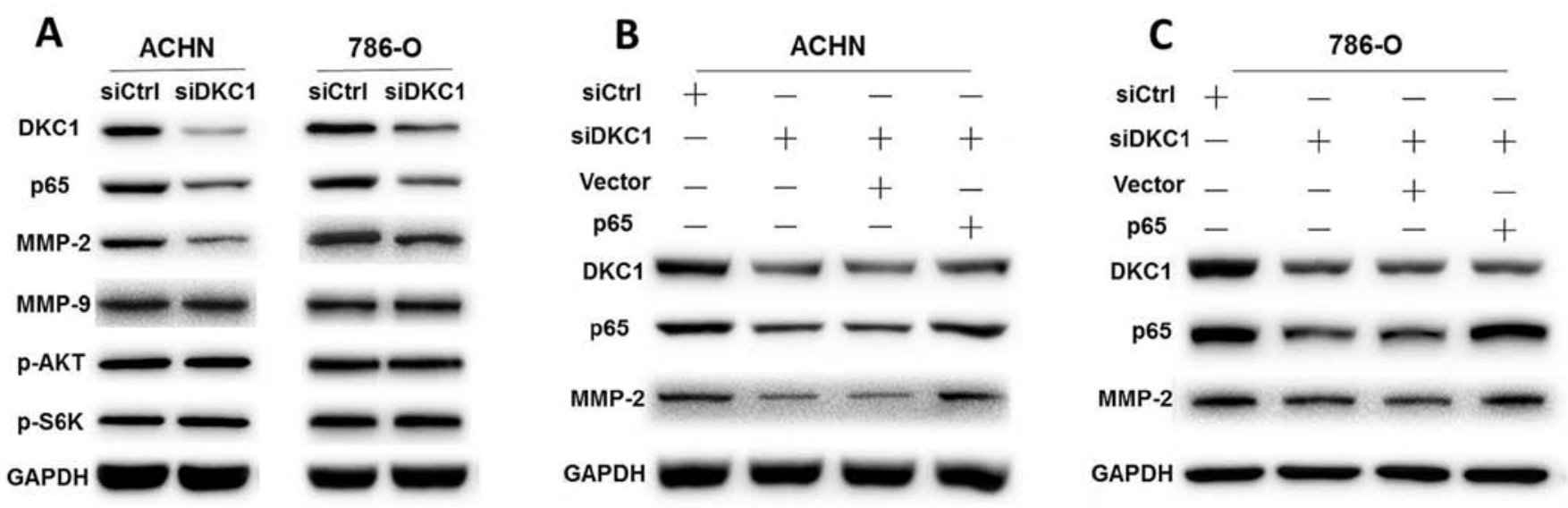

Figure 5. DKC1 regulates the expression of $\mathrm{p} 65$ and MMP-2. (A) Western blotting was performed to investigate the expression of cell proliferation and metastasis-related proteins, such as p65, MMP-2, MMP-9, p-AKT, p-S6K in ACHN and 786-O with different DKC1 expression levels. (B and C) Western blot analysis revealed that the p65 and MMP-2 expression in DKC1-knockdown ACHN and 786-O cells was significantly rescued by p65 expression plasmid when compared with the corresponding controls.

A

B

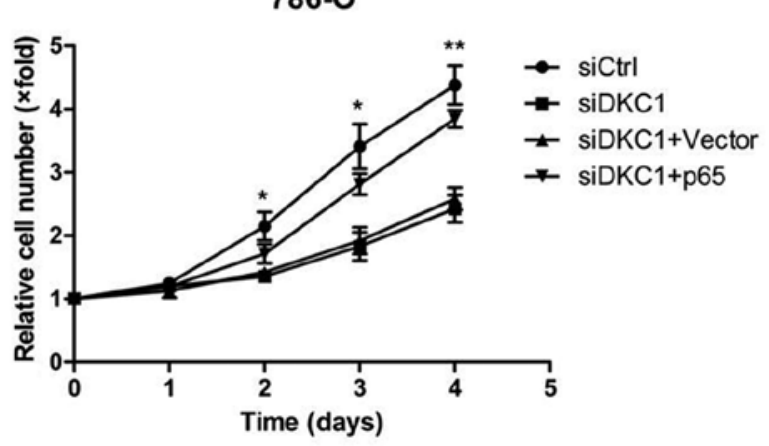

C

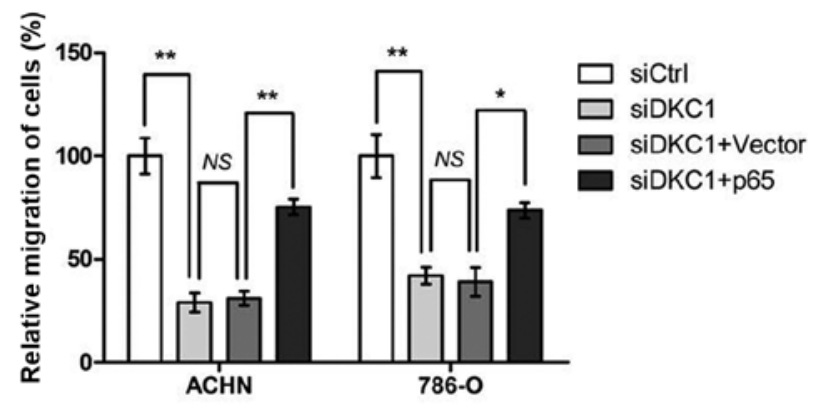

D

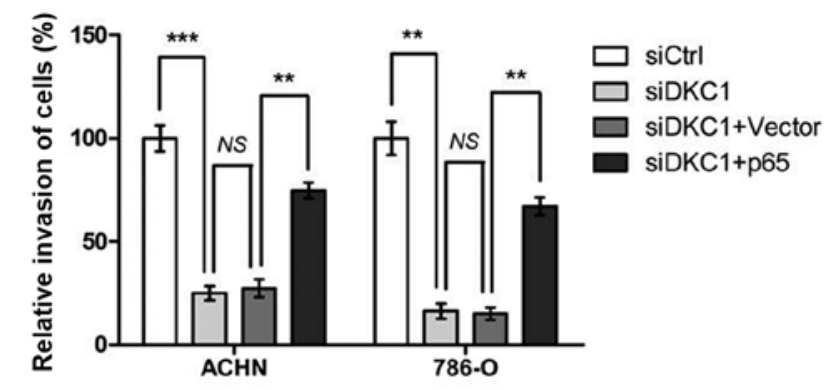

Figure 6. p65 rescues the decreased abilities of proliferation, migration and invasion of ccRCC cells induced by knockdown of DKC1. (A and B) p65 overexpression rescued the reduced abilities of proliferation in DKC1-knockdown ACHN and 786-O cells. (C and D) p65 overexpression rescued the reduced abilities of migration and invasion in DKC1-knockdown ACHN and 786-O cells. The cell migration and invasion number per field was counted in three random fields in ACHN and 786-O cells. Data are presented as the mean $\pm \mathrm{SD},{ }^{*} \mathrm{P}<0.05,{ }^{* *} \mathrm{P}<0.01,{ }^{* * *} \mathrm{P}<0.001$. ccRCC, clear cell renal cell carcinoma.

factor of cell proliferation and survival (17), we detected the expression levels of $\mathrm{p}-\mathrm{AKT}$ and $\mathrm{p}-\mathrm{S} 6 \mathrm{~K}$. However, the results revealed that the protein levels of $\mathrm{p}-\mathrm{AKT}$ and $\mathrm{p}-\mathrm{S} 6 \mathrm{~K}$ were similar regardless of the expression levels of DKC1 (Fig. 5A).

To further investigate whether DKC1 regulated ccRCC cell proliferation and metastasis though the NF- $\kappa$ B/MMP-2 signaling pathway, p65 rescue assays were performed. We co-transfected p65 overexpression plasmids and DKC1 siRNAs in ACHN and 786-O cells, respectively. Our data revealed that p65 overexpression significantly rescued the expression of MMP-2 (Fig. 5B and C). The cell proliferation assays revealed that the decreased abilities of cell proliferation in the DKC1-knockdown ACHN and 786-O cells could be markedly accelerated by p65 overexpression (Fig. 6A and B). 
A
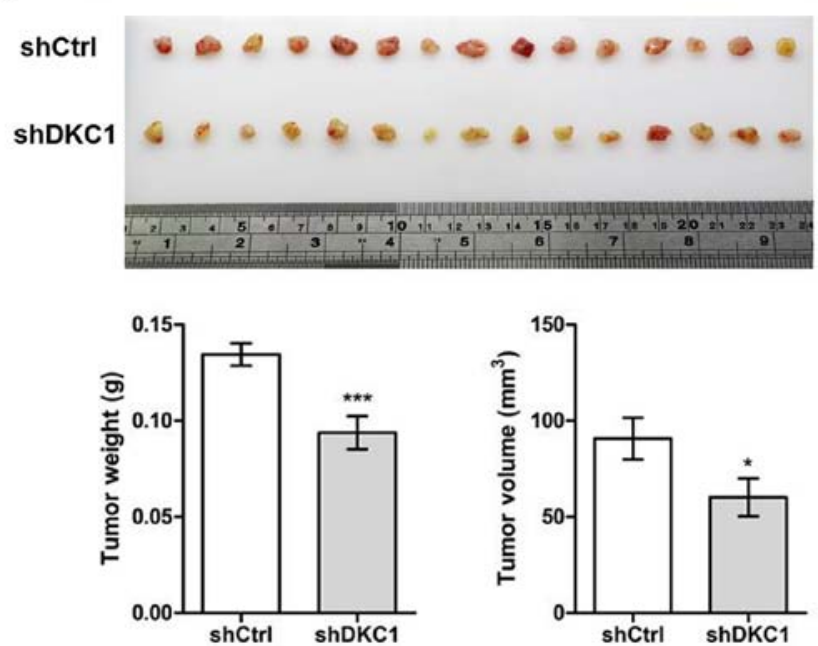

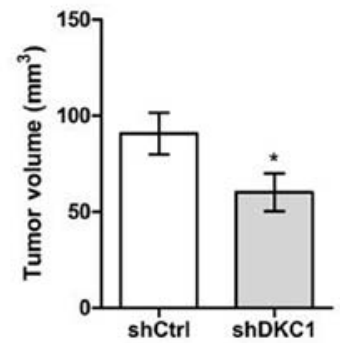

B

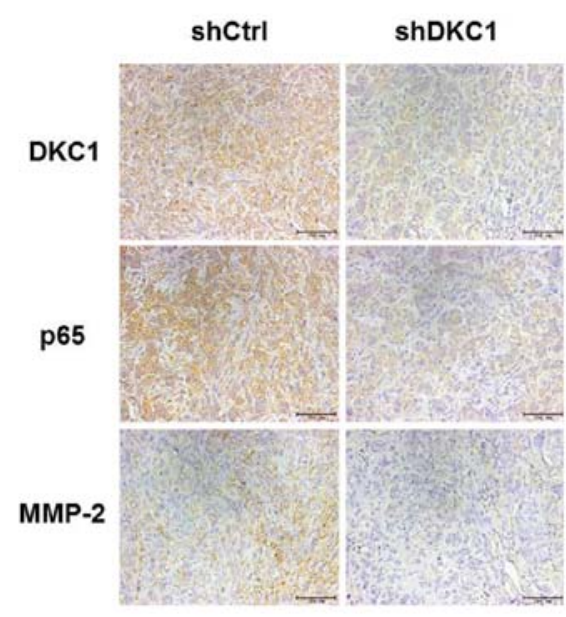

C
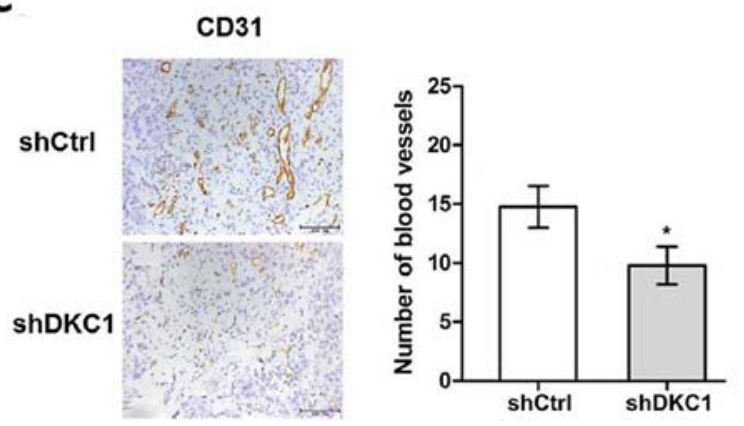

Figure 7. DKC1 promotes proliferation and angiogenesis of ccRCC in vivo. (A) Images of subcutaneous tumors resected from mice after 2 weeks of growth in vivo. (B) Immunostaining of DKC1, p65 and MMP-2 in subcutaneous tumors of DKC1 ${ }^{\mathrm{KD}}$ and control ACHN groups. DKC1, p65 and MMP-2 expression in $\mathrm{DKC} 1^{\mathrm{KD}}$ group were much lower than the control group. (C) Immunostaining of CD31 in subcutaneous tumors of DKC1 ${ }^{\mathrm{KD}}$ and control $\mathrm{ACHN}$ groups and the number of blood vessels marked by CD31 per field was counted in three random fields. Data are presented as the mean $\pm \mathrm{SD},{ }^{*} \mathrm{P}<0.05,{ }^{* * *} \mathrm{P}<0.001 . \mathrm{ccRCC}$ clear cell renal cell carcinoma.

Moreover, the results of cell migration and invasion revealed that $\mathrm{p} 65$ overexpression markedly rescued the DKC1-knockdown-decreased cell migration and invasion abilities (Fig. 6C and D). These results indicated that p65 and MMP-2 functioned as the downstream targets of DKC1 in ccRCC cell proliferation, migration and invasion.

DKCl accelerates the proliferation and angiogenesis of ccRCC cells in vivo. To further validate the function of DKC1 in the regulation of ccRCC proliferation in vivo, $\mathrm{DKC} 1^{\mathrm{KD}}$ - $\mathrm{ACHN}$ cell lines and Ctrl-ACHN cell lines were established. The stable DKC1 knockdown and control ACHN cells were subcutaneously injected into BALB/c nude mice. After 2 weeks, the two groups of mice were sacrificed and their subcutaneous tumors were excised (Fig. 7A) and statistical analysis was performed on the differences in tumor weight and volume between the two groups of mice. Our data revealed that a significant decrease of tumor weight and volume was observed in the $\mathrm{DKC} 1^{\mathrm{KD}}$ group when compared with the control group (Fig. 7A). Then, we carried out immunohistochemical staining on paraffin-sectioned neoplastic tissues. The data revealed that the expression of DKC1, p65 and MMP-2 were markedly reduced in the knockdown group compared with the control group (Fig. 7B). In addition, the visual examination revealed that neovessels in the subcutaneous tumors were reduced in the $\mathrm{DKCl}^{\mathrm{KD}}$ group when compared with those in the control group (Fig. 7A). Previous research had demonstrated that NF- $\mathrm{KB}$ promoted angiogenesis (18). To further validate the difference of angiopoiesis between the two groups, we detected the expression of CD31 in the two groups. The results revealed that the number of vessels labeled by CD31 were significantly fewer in the $\mathrm{DKCl}^{\mathrm{KD}}$ group than the control group (Fig. 7C). These results further confirmed our conclusion which had been previously demonstrated in vitro.

\section{Discussion}

Updated research has demonstrated that functional genes which play a role in tumorigenesis can be regarded as potential biomarkers for the diagnosis and prognosis of ccRCC patients (19). DKC1, an X-linked gene encoding dyskerin at $\mathrm{Xq} 28$, is a crucial ingredient of the telomerase complex and is indispensable for normal telomere function and the posttranscriptional modification of precursor rRNA $(20,21)$. It has been revealed to exert diverse biological functions and have prognostic value in numerous types of cancer (22-24). In the 
present study, we examined the prognostic value and biological role of $\mathrm{DKC1}$ in ccRCC. Using retrospective cohorts of ccRCC patients with TMAs, we discovered that DKC1 expression was significantly upregulated in ccRCC compared with normal renal tissues, and positively associated with TNM stage. Moreover, ccRCC patients with increased DKC1 expression had an unfavorable survival, and multivariate COX regression analysis revealed that positive $\mathrm{DKC1}$ expression was an independent hazardous indicator for the prognosis of ccRCC patients. Our findings from survival analysis conformed with the data obtained in The Cancer Genome Atlas (TCGA) collected from PROGgeneV2 platform. These results revealed that $\mathrm{DKC1}$ was a prognostic factor for ccRCC patients and may act as an oncogene in ccRCC progression.

RCC is the predominant malignancy of kidney cancer and is greatly resistant to chemotherapy and radiation. The status of the p53 tumor-suppressor gene has been associated with the efficacy of chemotherapy and radiation, where aberrant p53 function can be attributed to defective responsiveness to treatment $(25,26)$. However, p53 is rarely mutated in RCC, which suggests that other genes may be involved in the regulation of tumorigenesis in RCC (27). To date, few studies have reported the potential role of $\mathrm{DKC} 1$ in tumorigenesis and these results are disputable. von Stedingk et al (28) revealed that a potential function of DKC1 was to increase telomerase activity and contribute to advanced tumors, suggesting an oncogenic role of DKC1. However, Montanaro et al (29) reported that low $\mathrm{DKC1}$ expression was correlated with tumor progression, thus revealing that $\mathrm{DKC1}$ may serve as a tumor suppressor. The molecular mechanism and significance of DKC1 in ccRCC progression are still unclear. Our results demonstrated that DKC1 expression tends to be associated with adverse clinicopathological characteristics in ccRCC patients, which supports its role in tumor promotion.

Tumor development and progression require six necessary changes to normal cell physiology: An independent growth signaling pathway; sustained angiogenesis; resistance to growth inhibition; escape of apoptosis; tissue invasion and metastasis; and cell immortality (30). In the present study, we investigated the role of $\mathrm{DKC} 1$ in several important processes, such as proliferation, migration, invasion and angiogenesis. Our data revealed that DKC1 expression regulated ccRCC cell proliferation in vitro and in vivo, which was consistent with our clinical data that DKC1 expression was associated with tumor diameter. Moreover, we demonstrated that knockdown of DKC1 markedly inhibited ccRCC cell migration and invasion in vitro and angiogenesis in vivo. These findings could explain our previous assertion that positive DKC1 expression was associated with enhanced lymph node metastases.

Nuclear factor of $\kappa \mathrm{B}(\mathrm{NF}-\kappa \mathrm{B})$ is a transcriptional factor and it has been most extensively studied for its function in immunity and inflammation (31). Until recent decades, $N F-\kappa B$ has been characterized by its critical role in cancer development and progression and induces the expression of MMPs $(32,33)$. Matrix metalloproteinases (MMPs) can break down the extracellular matrix (ECM) in numerous malignant tumors and are crucial for metastasis-promoting genes (34). Except for their role in the extracellular matrix and migration of cancer cells, MMPs also regulate signaling pathways which control cell growth and angiogenesis (35).
In the present study, we investigated whether DKC1 regulated ccRCC proliferation and migration via NF- $\kappa \mathrm{B}$ and MMPs, and we demonstrated that knockdown of DKC1 significantly inhibited the protein expression of p65 and MMP-2, which may well account for our proliferation, migration and invasion results. However, in our study, MMP-9 expression did not appear to be activated by p65 in ccRCC cells. The functions of p65 and MMP-2 in DKC1-mediated ccRCC cell proliferation, migration and invasion were further explored by co-transfection of both DKC1 siRNAs and p65 overexpression plasmids, which rescued the decreased cell proliferation, migration and invasion after DKC1 knockdown. Moreover, our subcutaneous tumor model in vivo revealed that knockdown of DKC1 significantly suppressed proliferation and angiogenesis of tumors and the expression of p65, MMP-2 and CD31 in tumor tissues, which conformed to the in vitro results and cohort of ccRCC patients. Unfortunately, we did not explore the potential mechanism between $\mathrm{DKC} 1$ and the $\mathrm{NF}-\kappa \mathrm{B}$ pathway, and therefore whether the regulation of $\mathrm{NF}-\kappa \mathrm{B}$ by $\mathrm{DKC1}$ is direct or indirect is not clear. However, we did determine a possible relationship between them. Recently, it has been established that DKC1 is a direct target of c-Myc in several types of cancer (36). Thus, we speculated that NF- $\kappa \mathrm{B}$ could also directly activate $\mathrm{DKC1}$ expression, and p65 was regulated by $\mathrm{DKC1}$ via negative feedback. In future research, we will explore the potential molecular mechanisms between DKC1 and the NF- $\kappa \mathrm{B}$ signaling pathway in ccRCC cells consistently.

In conclusion, DKC1 expression was markedly enhanced in ccRCC compared with normal renal tissues. Positive DKC1 expression in ccRCC tissues was markedly associated with unfavorable clinicopathological characteristics and a dismal prognosis of patients, which can to some extent be explained by the NF- $\mathrm{B} / \mathrm{MMP}-2$ signaling pathway which regulated proliferation, migration, invasion and angiogenesis of ccRCC cells. Therefore, these results demonstrated that DKC1 may act as a significant prognostic indicator and therapeutic target for ccRCC.

\section{Acknowledgements}

Not applicable.

\section{Funding}

The present study was funded by grants from the National Natural Science Foundation of China (nos. 81472663, 81502280 and 81672845), the Education Department of Jiangsu Province (no. 15KJA320006) and the Project of Invigorating Health Care through Science, Technology and Education from Jiangsu Province.

\section{Availability of data and materials}

The datasets used during the present study are available from the corresponding author upon reasonable request.

\section{Authors' contributions}

MZ, JB and YP conceived and designed the experiments; YP, $\mathrm{RJ}$ and FC conducted the experiments; MZ and PH carried 
out the statistical analysis; HS and TJ supported the experiments and helped to draft the manuscript. All authors have read and approved the manuscript and agree to be accountable for all aspects of the research in ensuring that the accuracy or integrity of any part of the work are appropriately investigated and resolved.

\section{Ethics approval and consent to participate}

Informed consents from all patients were obtained and institutional approval was obtained by the Review Board of the Affiliated Hospital of Xuzhou Medical University prior to this study. The animal studies were approved by the Animal Care Committee of Xuzhou Medical University.

\section{Patient consent for publication}

Not applicable.

\section{Competing interests}

The authors declare that they have no competing interests.

\section{References}

1. Volpe A and Patard JJ: Prognostic factors in renal cell carcinoma. World J Urol 28: 319-327, 2010.

2. Rini BI, Campbell SC and Escudier B: Renal cell carcinoma. Lancet 373: 1119-1132, 2009

3. Lu X, Gu W, Zhang H, Zhu Y, Shi G and Ye D: Oligometastatic state predicts a favorable outcome for renal cell carcinoma patients with bone metastasis under the treatment of sunitinib. Oncotarget 7: 26879-26887, 2016.

4. Singer EA, Gupta GN and Srinivasan R: Update on targeted therapies for clear cell renal cell carcinoma. Curr Opin Oncol 23 283-289, 2011.

5. Heiss NS, Knight SW, Vulliamy TJ, Klauck SM, Wiemann S, Mason PJ, Poustka A and Dokal I: X-linked dyskeratosis congenita is caused by mutations in a highly conserved gene with putative nucleolar functions. Nat Genet 19: 32-38, 1998.

6. Kirwan M and Dokal I: Dyskeratosis congenita: A genetic disorder of many faces. Clin Genet 73: 103-112, 2008.

7. Sieron P, Hader C, Hatina J, Engers R, Wlazlinski A, Müller M and Schulz WA: DKC1 overexpression associated with prostate cancer progression. Br J Cancer 101: 1410-1416, 2009.

8. O'Brien R, Tran SL, Maritz MF, Liu B, Kong CF, Purgato S, Yang C, Murray J, Russell AJ, Flemming CL, et al: MYC-Driven neuroblastomas are addicted to a telomerase-independent function of dyskerin. Cancer Res 76: 3604-3617, 2016.

9. Liu B, Zhang JL, Huang C and Liu H: Dyskerin overexpression in human hepatocellular carcinoma is associated with advanced clinical stage and poor patient prognosis. PloS one 7: e43147, 2012.

10. Bellodi C, Krasnykh O, Haynes N, Theodoropoulou M, Peng G, Montanaro L and Ruggero D: Loss of function of the tumor suppressor DKC1 perturbs p27 translation control and contributes to pituitary tumorigenesis. Cancer Res 70: 6026-6035, 2010.

11. Montanaro L, Calienni M, Bertoni S, Rocchi L, Sansone P, Storci G, Santini D, Ceccarelli C, Taffurelli M, Carnicelli D, et al: Novel dyskerin-mediated mechanism of p53 inactivation through defective mRNA translation. Cancer Res 70: 4767-4777, 2010.

12. Mei P, Bai J, Shi M, Liu Q, Li Z, Fan Y and Zheng J: BRMS1 suppresses glioma progression by regulating invasion, migration and adhesion of glioma cells. PloS One 9: e98544, 2014.

13. Bai J, Yong HM, Chen FF, Song WB, Li C, Liu H and Zheng JN: RUNX3 is a prognostic marker and potential therapeutic target in human breast cancer. J Cancer Res Clin Oncol 139: 1813-1823, 2013.

14. Bai J, Zhou Y, Chen G, Zeng J, Ding J, Tan Y, Zhou J and Li G: Overexpression of Cullin1 is associated with poor prognosis of patients with gastric cancer. Hum Pathol 42: 375-383, 2011.
15. Karin M: Nuclear factor-kappaB in cancer development and progression. Nature 441: 431-436, 2006

16. Takeshita H, Yoshizaki T, Miller WE, Sato H, Furukawa M, Pagano JS and Raab-Traub N: Matrix metalloproteinase 9 expression is induced by Epstein-Barr virus latent membrane protein $1 \mathrm{C}$-terminal activation regions 1 and 2 . J Virol 73: 5548-5555, 1999.

17. Cully M, You H, Levine AJ and Mak TW: Beyond PTEN mutations: The PI3K pathway as an integrator of multiple inputs during tumorigenesis. Nat Rev Cancer 6: 184-192, 2006.

18. Huang S, Robinson JB, Deguzman A, Bucana CD and Fidler IJ: Blockade of nuclear factor-kappaB signaling inhibits angiogenesis and tumorigenicity of human ovarian cancer cells by suppressing expression of vascular endothelial growth factor and interleukin 8. Cancer Res 60: 5334-5339, 2000.

19. Audenet F, Yates DR, Cancel-Tassin G, Cussenot O and Roupret M: Genetic pathways involved in carcinogenesis of clear cell renal cell carcinoma: Genomics towards personalized medicine. BJU Int 109: 1864-1870, 2012.

20. Mochizuki Y, He J, Kulkarni S, Bessler M and Mason PJ: Mouse dyskerin mutations affect accumulation of telomerase RNA and small nucleolar RNA, telomerase activity, and ribosomal RNA processing. Proc Natl Acad Sci USA 101: 10756-10761, 2004.

21. Filipowicz W and Pogacic V: Biogenesis of small nucleolar ribonucleoproteins. Curr Opin Cell Boil 14: 319-327, 2002.

22. Westermann F, Henrich KO, Wei JS, Lutz W, Fischer M, König R, Wiedemeyer R, Ehemann V, Brors B, Ernestus K, et al: High Skp2 expression characterizes high-risk neuroblastomas independent of MYCN status. Clin Cancer Res 13: 4695-4703, 2007.

23. Schaner ME, Ross DT, Ciaravino G, Sorlie T, Troyanskaya O, Diehn M, Wang YC, Duran GE, Sikic TL, Caldeira S, et al: Gene expression patterns in ovarian carcinomas. Mol Boil Cell 14: 4376-4386, 2003.

24. Poncet D, Belleville A, t'kint de Roodenbeke C, Roborel de Climens A, Ben Simon E, Merle-Beral H, Callet-Bauchu E, Salles G, Sabatier L, Delic J and Gilson E: Changes in the expression of telomere maintenance genes suggest global telomere dysfunction in B-chronic lymphocytic leukemia. Blood 111: 2388-2391, 2008.

25. Lowe SW: Cancer therapy and p53. Curr Opin Oncol 7: 547-553, 1995.

26. Lowe SW, Bodis S, McClatchey A, Remington L, Ruley HE, Fisher DE, Housman DE and Jacks T: p53 status and the efficacy of cancer therapy in vivo. Science 266: 807-810, 1994.

27. Tomasino RM, Morello V, Tralongo V, Nagar C, Nuara R, Daniele E, Curti M and Orestano F: p53 expression in human renal cell carcinoma: An immunohistochemical study and a literature outline of the cytogenetic characterization. Pathologica 86: 227-233, 1994.

28. von Stedingk K, Koster J, Piqueras M, Noguera R, Navarro S, Påhlman S, Versteeg R, Ora I, Gisselsson D, Lindgren D and Axelson H: snoRNPs regulate telomerase activity in neuroblastoma and are associated with poor prognosis. Transl Oncol 6: 447-457, 2013.

29. Montanaro L, Brigotti M, Clohessy J, Barbieri S, Ceccarelli C, Santini D, Taffurelli M, Calienni M, Teruya-Feldstein J, Trerè D, et al: Dyskerin expression influences the level of ribosomal RNA pseudo-uridylation and telomerase RNA component in human breast cancer. J Pathol 210: 10-18, 2006.

30. Hanahan D and Weinberg RA: The hallmarks of cancer. Cell 100: 57-70, 2000.

31. Karin M, Cao Y, Greten FR and Li ZW: NF-kappaB in cancer: From innocent bystander to major culprit. Nat Rev Cancer 2: 301-310, 2002.

32. Uhlik M, Good L, Xiao G, Harhaj EW, Zandi E, Karin M and Sun SC: NF-kappaB-inducing kinase and IkappaB kinase participate in human T-cell leukemia virus I Tax-mediated NF-kappaB activation. J Biol Chem 273: 21132-21136, 1998.

33. Kumar A, Takada Y, Boriek AM and Aggarwal BB: Nuclear factor-kappaB: Its role in health and disease. J Mol Med (Berl) 82: 434-448, 2004.

34. Kim A, Kim MJ, Yang Y, Kim JW, Yeom YI and Lim JS: Suppression of NF-kappaB activity by NDRG2 expression attenuates the invasive potential of highly malignant tumor cells. Carcinogenesis 30: 927-936, 2009.

35. Kessenbrock K, Plaks V and Werb Z: Matrix metalloproteinases: Regulators of the tumor microenvironment. Cell 141: 52-67, 2010.

36. Alawi $\mathrm{F}$ and Lee $\mathrm{MN}$ : $\mathrm{DKC} 1$ is a direct and conserved transcriptional target of c-MYC. Biochem Biophys Res Commun 362: 893-898, 2007. 